but not $50 S$ subunits is the same as in wild type strains. In other words, it is the $30 S$ subunits and other components necessary for initiation and not the $70 \mathrm{~S}$ ribosome which affect $m$ RNA synthesis.

\title{
GENE CONTROL
}

\section{Histones-Animal and Vegetable}

from our Molecular Biology Correspondent

THE biochemical world has long been divided on the question of whether the histones are actively involved in suppression and activation of genes, or whether they exist as essentially passive-and therefore, according to one view, slightly dreary-packaging materials. Among the indications that the role of the histones is not purely passive are the many observations of the enzymic modification of certain of their side chains under circumstances which suggest that this may somehow be involved in the control of transcription of the DNA. The attachment of acetyl groups to the N-termini of two of the histones, and of methyl and phosphoryl groups to side chains of others, has been demonstrated. Allfrey and his co-workers found that whereas some of these modifying groups remain more or less permanently bound, others are metabolically active, in the sense that a radioactive precursor is rapidly incorporated at these points.

Gershey, Vidali and Allfrey (J. Biol. Chem., 243, 5018 ; 1968) have now found, from experiments involving pulse-labelling of calf thymus nuclei with ${ }^{14} \mathrm{C}$-acetate, that the histone component $f 2 a \mathrm{l}$ exhibits this type of rapid turnover. Moreover, proteolytic digestion of the histone and separation of the fragments led to the identification of the label in a single $\varepsilon$-acetyllysine residue. This is therefore the metabolically active site. Rapid labelling also occurs in the argininerich fraction, $f 3$.

Meanwhile, in E. L. Smith's laboratory in Los Angeles, the latter protein (also termed histone-IV in another system of nomenclature) has been completely sequenced, and the modified side chain located. This work, reported at the autumn meeting of the National Academy of Sciences, must surely be expected to stimulate a wide resurgence of interest in the histone field. The protein has 102 residues, the rapidly labelled site being lys-16. In the protein, as isolated, this residue is acetylated in half the molecules. There is also a fully methylated lysine at position 20 , the remaining nine lysines being unmodified. The sequence shows some curious features-in particular a marked polarization of charge, such that the N-terminal end contains a predominance of the positive groups, and the other end most of the negative and hydrophobic side chains. This suggests that the attachment to DNA may primarily involve one end of the molccule, perhaps leaving the other free to engage in some function which is as yet unknown.

The same workers (Delange, Fambrough, Smith and Bonner) also prepared the corresponding histone fraction from pea secdlings-an operation involving germination of no less than 20 tons of seeds-and now have the substantially complete sequence. An early report, in which the first 19 residues from the C-terminal end were found to be identical in the pea and calf thymus proteins, is borne out. Indeed, it appears that the chains are identical in length, and differ only by two replacements, one of arginine for lysine, the other of isoleucine for valine; in addition lys-20 is not methylated in the pea protein. Apart from this astonishing evolutionary conservation of sequencea phenomenon altogether unique among the very considerable number of known sequences in a range of proteins of the most diverse functions-this result points incluctably to some highly specific and precisely defined biochemical function for the histone.

\section{VIROLOGY \\ Coronaviruses}

A NEW group of viruses with the name of coronaviruses has been recognized by an informal group of virologists who have sent their conclusions to Nature. (They are J. D. Almeida; D. M. Berry; C. H. Cunningham; D. Hamre; M. S. Hofstad; L. Mallucci; K. MeIntosh; D. A. J. Tyrrell.)

They point out that with negative staining, avian infectious bronchitis virus has a characteristic electron microscopic appearance resembling, but distinct from, that of myxoviruses. Particles are more or less rounded in profile; although there is a certain amount of polymorphism, there is also a characteristic "fringe" of projections $200 \AA$ long, which are rounded or petal shaped, rather than sharp or pointed, as in the myxoviruses. This appearance, recalling the solar corona, is shared by mouse hepatitis virus and several viruses recently recovered from man, namely strain B814, 229E and several others. These viruses also share a number of other properties as indicated in the table. (Anyone interested in the data on which the table is based may obtain a short bibliography on application to Dr D. A. J. Tyrrell at the Common Cold Research Unit, Salisbury, Wiltshire.)

PROPERTIES OF THESE VIRUSES Avian

infectious Mouse Human bronchitis hepatitis strains

Size. Filtration Electron microscopy* $80-120 \mathrm{~m} \mu \quad 100 \mathrm{~m} \mu \quad 80^{--160 \mathrm{~m} \mu}$ Characteristic surface structure Essential lipid (ether lability) Apparent ribonucleic acid content + (unsusceptibility to DNA inhibitors) Density of infectious unit 1.18 Replication in cytoplasmic vesicles +

* Negative contrast technique-projections are included in the diameter of the particlc.

Some other relevant properties should be mentioned. There is an antigenic relationship between the human and murine strains, but none has been detected between avian strains and the others. A hacmagglutinin has been detected by certain workers using avian infectious bronchitis virus and also antigens separable from the virus particle, but these have so far not been recorded for the human or murine strains.

In the opinion of the eight virologists these viruses are members of a previously unrecognized group which they suggest should be called the coronaviruses, to recall the characteristic appearance by which these viruses are identified in the electron microscope.

These suggestions have been received by members of the Myxovirus Study Group (chairman, Professor A. P. Waterson) under the International Committee for the Nomenclature of Viruses (ICNV). The suggestions were found acceptable and are now to be considered by the Vertcbrate Virus Committee of the ICNV. 